\title{
Lignin-Treated-Trichloromethylsilane Sorbent for Oil Spill Cleanup
}

\author{
Azhar N. A.*, Rahman W.A.W., Adrus N., Majid R. A. \\ School of Chemical and Energy Engineering, Faculty of Engineering, Universiti Teknologi Malaysia, 81310 \\ Johor Bahru, Johor, Malaysia.
}

*Corresponding Authors: amalina88@yahoo.com

\section{Article History}

Received: May 7, 2018

Received in revised form: October 27, 2018

Accepted: November 10, 2018 Published Online: February 10, 2019

\begin{abstract}
The effects of physical and chemical treatments of lignin with trichloromethylsilane (TCMS) as potential oil-spilled absorbent for waste water were investigated. The lignin was extracted from oil palm empty fruit bunch (OPEFB) using sodium hydroxide $(\mathrm{NaOH})$ through soda process method. The lignin was treated with TCMS at various time reactions. The result revealed that over $94 \%$ reduction of hydroxyl $(\mathrm{OH})$ group was achieved when TCMS was used. Besides, the hydrophobicity behaviour was improved with time reaction with water contact angle from $0^{\circ}$ to $128^{\circ}$. Scanning electron microscopy (SEM) was conducted to examine the structure and morphology of treated lignin and leads to the presence of lumps and wavy on the fibre surface. In contrast, native lignin shows a smooth surface. The treated lignin can quickly absorb carotino oil with improvement in oil capacity compared with raw fiber, respectively. As such, good environmental friendly lignin-treated-TCMS (L-TCMS) can be considered as potential alternative in oil-spill clean-ups application.
\end{abstract}

Keywords: Lignin; trichloromethylsilane; hydrophobic; sorbent; oil spill

\subsection{INTRODUCTION}

The world is facing various types of pollutant loaded into marine environment such as sewage and garbage, detergent, oil and petroleum derivatrives, pesticides and many more. Spill statistics are collected by a number of agencies in any country. The oil spill in water bodies is mainly due to the human activities. Oil production related processes such as drilling, refining, and transporting or land activities such as farming and manufacturing are examples that cause an oil spill. Animals, plants, human and marine ecosystems are affected by the spill. As reported by the World Wide Fund for Nature (WWF) in 2009, oil and petroleum are the major concerns as an estimated 400,000 tons/year of spilled oil is involved around the world. The International Tanker Owners Pollution Federation Limited (ITOPF), an agency that runs a training and gives regular advice to the goverment and industry on pollution damage caused by spills of oil, chemicals and other hazardous substances has reported in September 2016, a serious oil spill accident in the Gulf of Mexico.

Approximately 5,500 tonnes of oil was burnt in the explosion which sparked a fire. In Hong Kong, at least 1,000 tonnes of palm oil stearin was leaked into waters near Hong Kong in earlier August 2017. The leakage was caused by the collision between two vessels near the Pearl river estuary in southern China.

Many methodologies can be adopted for the purpose of cleaning up oil spills such as oil booms, burning in-situ, skimming, using dispenser or manual labour [1]. Another effective method is using a sorbent. Synthetic fibres such as polyesters or polypropylene and natural fibres derived from plants or biomass agriculture such as cotton, kapok, bagasse or rice straw are used as sorbents [1-4]. The latter is more attractive due to their biodegradable natures, abundant and cheap.Lignin is another type of bio-waste originated from the paper-related industries. In the paper making process, the fibres are treated through the Kraft process to obtain cellulose while lignin and hemicelluloses are discarded [1].

Generally, plant cell wall material is composed of three important components which are cellulose, lignin, and hemicellulose. Lignin is a kind of renewable biomass resource and the second richest output of organic compound on earth after cellulose 
[5]. Lignin is a complex amorphous polymer of phenylpropane units, which are highly cross-linked to each other with a variety of different chemical bonds with range of $1000 \mathrm{~g} / \mathrm{mol}$ to $20,000 \mathrm{~g} / \mathrm{mol}$ of molecular masses [6]. Three-dimensional aromatic lignin molecular structure for softwood with phenyl propane units linked together by a variety of stable carbon-carbon and carbon-oxygen carbon (ether) linkages with phenolic and alcoholic hydroxyl groups. Typical lignin contains both hydrophilic phenolic/aliphatic hydroxyl groups and lipophilic carbon backbone, which suggests a special affinity to polar and nonpolar phases. However, a certain modification step is often necessary to control its amphiphilicity and solubility [7]. In previous research, Mousavioun and Doherty (2010) have analysed the elemental composition, functional group and molecular weight of the lignin. They found that the fractionation process separated lignin into individual molecular weights and there were resulting in differences in the phenolic hydroxyl, methoxyl composition and carboxylic acids. Besides that, they found out that the properties of material produced were depended on these structural properties [6].

Cheap adsorption process using agricultural waste products have been tested as potential absorbents including cotton balls, corncobs, meranti sawdust and many more. These products also have been as a potential low-cost absorbents. Thus, a sustainable approach of waste materials to be recycled into high value products should be explored. For example, Azhar et al., 2015 studied on lignin as a potential absorbent to absorb water up to $400 \%$ of its own weight when grafted with polycrylic acid [8]. Platcom Ventures Company has reported that Malaysia has 3.87 millon ha of land planted with oil palm, which provides large amounts of lignocellulosic waste, oil palm empty fruit bunches (OPEFB) estimated around 30mil tones/year. Lignin is a material that contains both hydrophobic and hydrophilic groups in their molecular structure. With a same research by Azhar et al., 2015 found lignin-grafted-polyacrylic acid has a potential to absorb oil for water waste treatment application. However, like other natural fibres, lignin must be treated to reduce its hydrophilic nature to produce an oil sorbent. Acetic anhydride [4] and stearic acid [2] are among the reagents used to impart hydrophobic character to natural fibres. In this research, trichloromethylsilane (TCMS) was selected to treat the lignin due to its ability to react with both hydroxyl groups of cellulose and water through vapour deposition method. The process is more simple and considered as green chemistry [9]. Hence, with a proper design, lignin can be tailored into an effective sorbent for cleaning up the oil spill. To date, TCMS has never been used to treat lignin. Besides, the oil sorption capacity was also investigated.

\subsection{METHODOLOGY}

\subsection{Lignin Extraction}

Lignin was extracted from OPEFB by using a soda process. OPEFB was collected from the palm oil mill in Mersing, Johor. OPEFB was cleaned and cut into small peces before cooked with diluted of $10 \%$ concentration of sodium hydroxide $(\mathrm{NaOH})$ with distilled water at $150^{\circ} \mathrm{C}$ for 2 hours. Fibrous materials were removed through a strainer and black liqour was collected. Later, the diluted $10 \%$ of sulphuric acid was slowly added into the black liquor to achieve $\mathrm{pH} 3$. The slurry was filtered through a mesh cloth and the residue (lignin) was collected. The lignin was washed with copious amount of distilled water until the $\mathrm{pH}$ became natural. Then, the solid lignin was oven dried overnight at $60^{\circ} \mathrm{C}$ prior to be kept in a desiccator. Sodium Hydroxide $98 \%$ concentration $\mathrm{NaOH}$ was purchased from AGC Chemical (Thailand) LTD. Sulphuric Acid (98\% purity) was obtained from RCI Labscan Limitted, Thailand while trichloromethylsilane (97\% purity) was purchased from Medigene Sdn. Bhd. All materials were used as received.

\subsection{Treatment of Lignin with TCMS via Vapour Deposition Method}

The treatment of lignin with TCMS using a vapour deposition method was adopted from the previous works [9,10]. About $0.2 \mathrm{~g}$ of dried solid lignin was filled into a non woven tea bag. The bag was hung at the top of the sealed flask containing 12 $\mathrm{ml}$ of TCMS. Later, the flask was placed in the reaction chamber at temperature of $60^{\circ} \mathrm{C}$. Tea bag containing lignin was exposed to TCMS vapour at various exposure times (1.5 mins (S1), 3 mins (S2), 4.5 mins (S3), 6 mins (S4) and 7.5 mins (S5). The obtained lignin-treated TCMS (L-TCMS) was blown with air and rinsed with a generous amount of tap water to remove excess reagent and hydrogen chloride produced during the reaction (as shown in Table 1).

Table 1 A reaction scheme of lignin and TCMS via vapour deposition method [9].

\begin{tabular}{|c|c|c|c|}
\hline \multirow{2}{*}{ ation } & \multirow{2}{*}{$\begin{array}{l}\text { Sample } \\
\text { Identific }\end{array}$} & \multicolumn{2}{|c|}{ Reaction conditions } \\
\hline & & $\begin{array}{l}\text { TCMS amount } \\
(\mathrm{mL})\end{array}$ & $\begin{array}{l}\text { Reaction Time } \\
\text { (min) }\end{array}$ \\
\hline & Lignin & - & - \\
\hline & S1 & 12 & 1.5 \\
\hline & S2 & 12 & 3 \\
\hline & S3 & 12 & 4.5 \\
\hline & S4 & 12 & 6 \\
\hline & S5 & 12 & 7 \\
\hline
\end{tabular}




\subsection{Characterizations of native lignin and lignin treated TCMS}

The degree of hydrophobicities of native lignin and L-TCMS was determined by using a contact angle measurement with a dynamic sessile drop technique. About $2 \mu \mathrm{L}$ of distilled water was dropped onto the sample using a micro-syringe at room temperature. An average of five readings of contact angles were taken for each sample. The droplet images were captured by a digital camera. The angle value that $>90^{\circ}$ indicates a hydrophobic character [11]. Surface morphologies of the samples were investigated using scanning electron microscopy (SEM). The samples were frozen with liquid nitrogen prior to fracture. The samples were sputtered-coating with gold and the fractured surfaces were examined at x2000 magnification. In order to analyse the chemical changes in the Lignin-treated TCMS sample, the disappearance of functional group hydroxyl (-OH_was monitored by using attenuated total reflectance (ATR) mode FTIR (Shimadzu, IR Tracer 100). The FTIR spectra were obtained in the $4 \mathrm{~cm}^{-1}$ resolution mode with 32 scans averaged for each sample between $4000 \mathrm{~cm}^{-1}$ to $500 \mathrm{~cm}^{-1}$ at room temperature. In order to measure the degree of conversion, the FTIR spectra were collected in absorbance mode. This is because the degree of conversion was calculated using following equation [12].

$$
\text { Degree of conversion }(\%)=\frac{\left(A_{0}-A_{t}\right)}{A_{0}} \times 100 \%
$$

Where $\mathrm{A}_{0}$ is the peak area of $3300 \mathrm{~cm}^{-1}-3500 \mathrm{~cm}^{-1}$, including the absorption peak at $3342 \mathrm{~cm}^{-1}$ before lignin undergo treatment and $A_{1}$ is the peak area at reaction time, $t$.

\subsection{Measurements of oil sorption capacity}

The oil sorption capacities of L-TCMS samples were measured as previous work by Oribayo et al. where the sample was drained into oil for 1 minute. About $0.2 \mathrm{~g}$ sample was immersed in a beaker containing palm oil at room temperature. The sample was taken out from the oil and placed on the wire mesh to remove the excess oil, at different immersion times. The oil sorption capacity (Q) of the sample was calculated using Equation 1 [13].

$$
Q=\frac{M t-M i}{M i}
$$

where $Q=$ oil sorption capacity of the sorbent

$M t=$ weight of wetted sorbent after draining $(\mathrm{g})$

$M i=$ initial weight of sorbent $(\mathrm{g})$

\subsection{RESULTS AND DISCUSSION}

\subsection{IR-Spectra}

Lignin significantly contains both hydrophobic and hydrophilic groups in their molecular structure. However, the hydrophilic or hydrophobic character depends the on the original reaction with a wide range of blends to be produced (Lignin). The focus of this study is the preparation of possible lignin to be superhydrophobic where lignin undergoes a treatment with TCMS. Infrared spectra of the L-TCMS samples were compared to the spectra of original sample to see an establishment of superhydrophobic lignin via chemical vapour deposition and influence of time reaction of 1.5 minute (S1), 3 minute (S2), 4.5 minute (S3), 6 minute (S4), 7.5 minute (S5). 


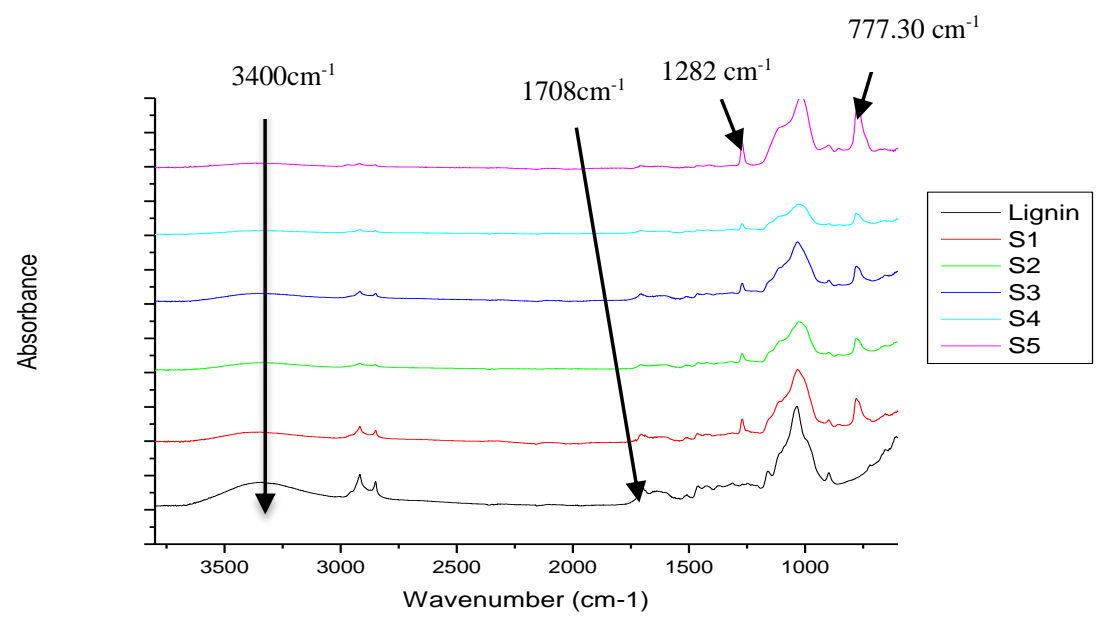

Figure 1. FTIR of lignin and L-TCMS.

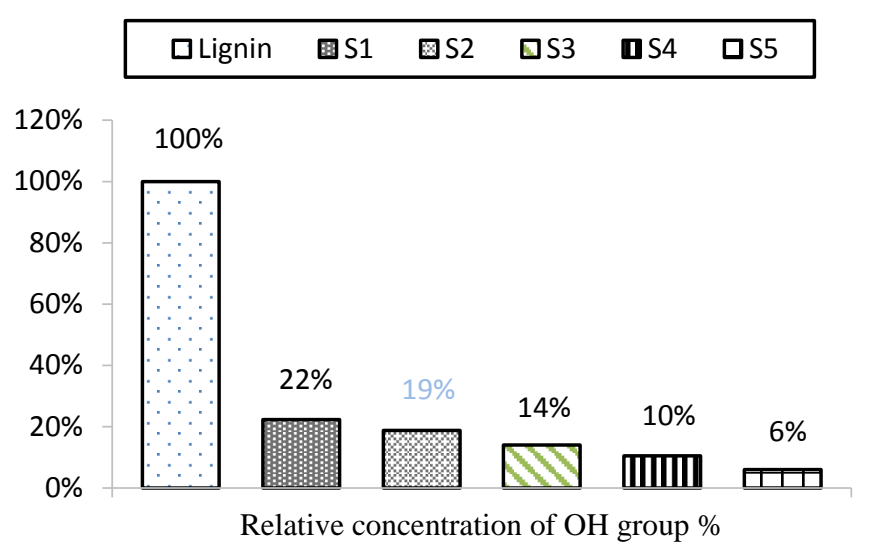

Figure 2. OH group remained after conversion for lignin and treated lignin.

Figure 1 shows a result of FTIR for each sample. A strong and broad absorption was observed at wavenumber of around 3300$3500 \mathrm{~cm}^{-1}$. This wavenumber was assigned to hydrogen bonded $(\mathrm{O}-\mathrm{H})$ stretching absorption. $\mathrm{O}-\mathrm{H}$ stretching region at a wavenumber of $3300-3500 \mathrm{~cm}^{-1}$ of native lignin of OPEFB spectra was more identical to the $\mathrm{O}-\mathrm{H}$ stretching region from sample 1 to sample 5. Meanwhile, the $\mathrm{O}-\mathrm{H}$ band in lignin treated (sample 1 to sample 5) seriously diminished the reaction products compared to native lignin, confirming that $\mathrm{OH}$ group in lignin was removed by the reaction with TCMS that almost instantly reacts with air moisture and hydroxyl $(-\mathrm{OH})$ groups in lignin to form siloxane and hydrogen chloride gas via vapour deposition method. The relative concentration of $\mathrm{OH}$ group was removed up to $94 \%$ from native lignin as shown in figure 2 . The relative band-height at this region decreased as time reaction of treated lignin is increased. Besides the bands characteristic of $\mathrm{OH}$ group and $\mathrm{CH}$ group, the presence of siloxane characteristic bands may be observed in the reaction products at $777.30 \mathrm{~cm}^{-1}$ belong to $\mathrm{CH}_{3}$ rocking and $\mathrm{Si}-\mathrm{CH}$ symetric stretching in $\mathrm{SiCH}_{3}$ and also can be seen that the peaks intensity of symmetric $\mathrm{CH}_{3}$ in $\mathrm{Si}-\mathrm{CH}_{3}$ at $1282 \mathrm{~cm}^{-1}$. The peak between $1020-1100 \mathrm{~cm}^{-1}$ should indicates the presence of Si-O-Si bond of the siloxane components which form from the covalent attachment of TCMS onto lignin. However, there are no significant changes in the wavenumber since the siloxane peak was overlaping with the $\mathrm{C}-\mathrm{O}$ stretching band in lignin as described by $\mathrm{Li}$ et al., 2007 and Cunha et al., 2010 where they studied the hydrophobicity changes of cellulose when treated to TCMS [9,14]. The most important evidence for the formation of new products is the precense of the $\mathrm{SiCH}_{3}$ absorption band, together with the disappearance of the carboxylic acid band rercorded in the starting disiloxanes at $1708 \mathrm{~cm}^{-1}$ [15].

\subsection{Water Contact Angle}

The surface wettability of the as-prepared substrates has been assessed by a series of WCA measurements. The WCA of the starting lignin fibre is $0^{\circ}$, classified as superhydrophilic material (when contact angle is less than $90^{\circ}$ ) where water rapidly 
sinks into the fibre due to the $-\mathrm{OH}$ group naturally in lignin . Treating the lignin with TCMS has increased hydrophobicity of lignin as reported in previous research by Tejado et. al., 2014 where TCMS reacts with air moisture and hydroxyl (-OH) group in lignin to form siloxane and it is also believed that the hydrogen chloride gas was vaporized to the air during chemical vapour deposition process [14]. As anticipated, the introduction of TCMS absorbed into the substrate results in the transformation of hydrophilic to hydrophobic character, with a WCA of $127.3^{\circ}$ obtained under optimal conditions. An examination of the effect of the reaction time on the surface wettability shows that the WCA of the resulting material increased with reaction time up to 7 min under comparable conditions. Figure 3 shows 5 readings of each sample and the final average. The contact angle of treated lignin S1 started shows hydrophobic behaviour initially and gradually increased till sample S5.

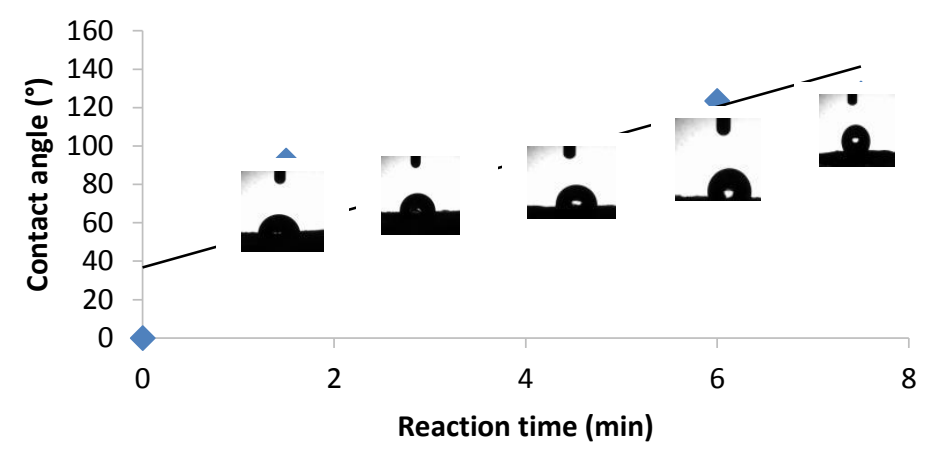

Figure 3. Effect of reaction time on the hydrophobic property of treated lignin with TCMS

\subsection{Scanning Electron Microscope (SEM)}

The surface properties of fibril lignin and treated lignin giving an interesting information for coating silane to the lignin. Treated lignin gave a rough surface. Besides that, the samples show the presence of lumps and waves with small pores revealing the presence of silica embedded in the polymer network as shown in figure 4 (b) and (c), respectively. The lumpy, wavy and rough surface became more pronounced as reaction time increased and at the same time, the capability of treated lignin enhanced the chances to repel water is very high which affected hydrophobicity the of L-TCMS.

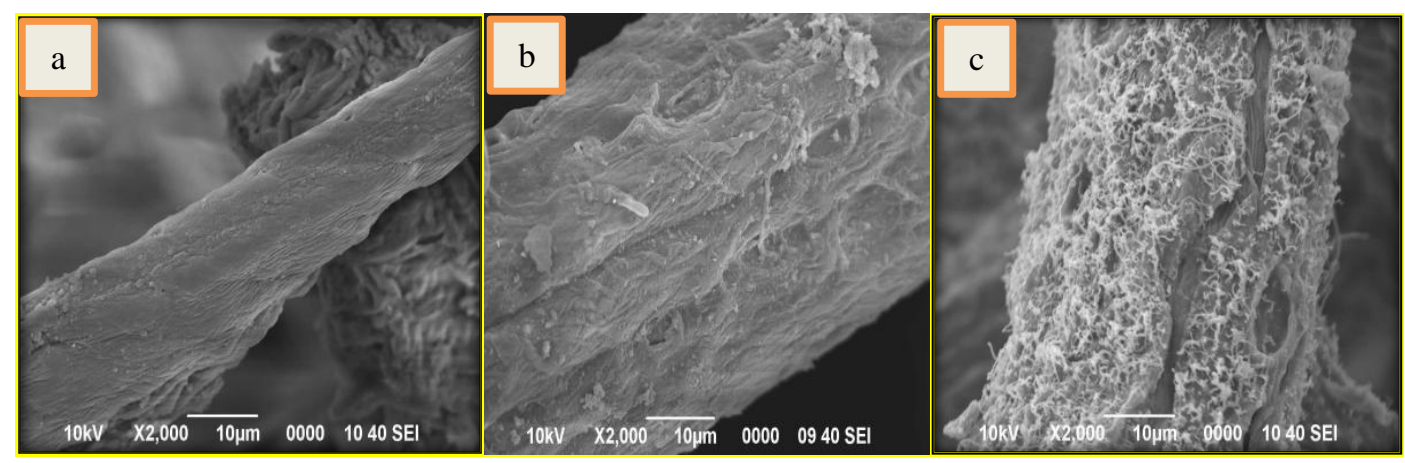

Figure 4. Surface morphology at x2000 magnification of a) native lignin, b) 3 minute time reaction (S2) c) 7.5 minute time reaction $(\mathrm{S} 5)$

\subsection{Oil Absorption Capacity}

To further investigate the oil absorption capacity properties of treated lignin for potential applications of oil spill clean-up, a sample was dipped into carotino oil. Oil absorption in the native lignin and treated lignin with different time reaction is presented in the table 2 . It can be seen that when lignin was introduced onto lignin surface, the lipophilic properties of lignin was increased up to $25 \%$, owing to bipolar properties of lignin [7]. In order to increase the lipophilic properties, the time reaction of lignin exposed to silane is increased to 7 minutes (sample S5) where sample S5 exhibited the highest oil sorption capacity i.e. $70 \%$ with respect to untreated lignin. It is obvious that there is clear correlation between volume of absorbed oil and time reaction applied in preparation of treated lignin [16]. With an appropriate coated silicon surface morphology and inherent hydrophobicity and olephilicity, L-TCMS appeares to be a potential sorbent material in oil spill clean up application [13]. 
Azhar N. A. et al. /JEST-Joumal of Energy and Safety Technology. vol. 1, no. 2-2 (2019): 43 - 49

Table 2. Oil absorption capacity of native lignin and treated lignin-TCMS

\begin{tabular}{|c|c|c|c|c|}
\hline Sample & Before $(\mathrm{g})$ & After $(\mathrm{g})$ & Oil absorption capacity (\%) & Stdev \\
\hline Lignin & 0.2 & 0.2498 & 24.90 & 0.73 \\
\hline S1 & 0.2 & 0.2626 & 31.30 & 0.27 \\
\hline S2 & 0.2 & 0.2634 & 31.70 & 0.29 \\
\hline S3 & 0.2 & 0.3057 & 52.85 & 0.63 \\
\hline S4 & 0.2 & 0.3078 & 53.90 & 0.59 \\
\hline S5 & 0.2 & 0.34 & 70.00 & 0.57 \\
\hline
\end{tabular}

\subsection{CONCLUSION}

In this study, a hydrophobic and oleophilic properties of lignin-treated-TCMS was successfully obtained via a chemical vapour deposition method for the cleanup and recovery of spilled oil in water waste treatment. This was achieved when silica group (-Si) was coated onto the lignin fiber and the oil can be quickly absorbed by L-TCMS as recorded from Sample S1 to sample S5 up to $70 \%$ with respect to untreated lignin. The L-TCMS which was based on low-cost materials, straight foward preparation and easy to be treated, exhibited a good balance of hydrophobic-oleophilic surface property which is an important character for good oil sorbent, may be a promising substitute for oil-absorbing fiber used in the clean-up of oil spills in water bodies.

Work is in progress to extend the scope of this preliminary study to grafting/coating with the monomer as a binder and other reactions in order to optimize the applications and enhance the hidrophobicity effects, while maintaining the original simplicity and sustainability. For example in further study by using polystyrene to hold the lignin since lignin was produced in powder form. In the same time, the superoleophilicity of lignin will be expected [17].

\section{Acknowledgements}

The authors would like to thank Universiti Teknologi Malaysia for furbishing the grant (GUP) to expedite the research work [vot: Q.J130000.2546.14H38]. Last, but not least, research colleagues for all the assistance granted throughout the research period.

\section{References}

[1] Lim T. T., Huang X. 2007. Evaluation of Hydrophobicity/Oleophilicity of Kapok and Its Performance in Oily Water Filtration: Comparison of Raw and Solvent-treated Fibers. Industrial Crops and Products. 26: 125-134.

[2] Hoai N. T., Sang N. N., Hoang T. D. 2016. Oil Spill Cleanup Using Stearic-acid-modified Natural Cotton. Journal of Materials and Environment Science. 7 (7): 2498-2504

[3] Behnood R., Anvaripour B., Jaafarzeadeh N., Farasati M. 2016. Oil Spill Sorption Using Raw and Acetylated Sugarcane Bagasse. Journal of Central South University. 23: 1618-1625.

[4] Sun X. F., Sun R., Sun J. X., 2002. Acetylation of Rice Straw With or Without Catalysts and Its Characterization as a Natural Sorbent in Oil Spill Cleanup. Journal of Agricultural and Food Chemistry. 50: 6428-6433.

[5] W.G. Glasser and S.S. Kelly. 1987. Light Stability of Polymers. Encyclopedia of Polymer Science and Engineering. 795-852.

[6] Doherty W. O.S., Mousavioun P., Fellows C. M. 2010. Value-adding to Cellulosic Ethanol: Lignin Polymers. Industrial Crops and Products. $33(2): 259-276$.

[7] Zhang Z., Zhang Y., Lin Z., Mulyadi A., Mu W., Deng Y. 2017. Butryric Anhydride Modified Lignin and Its Oil-water Interfacial Properties. Chemical Engineering Science. 165: 55-64.

[8] Azhar N. A., Soloi S., Majid R. A., Jamaluddin J. 2015. Grafting Efficiency of Lignin-grafted-polyacrylic Acid. Applied Mechanics and Materials. 735: $182-185$

[9] Cunha A. G., Freire C., Silvestre A., Neto C. P., Gandini A., Belgacem M. N., Chaussy D., Beneventi D. 2010. Preparation of Highly Hydrophobic and Lipophobic Cellulose Fibers by A Straightforward Gas-solid Reaction. Journal of Colloid Interface Science. 344(2): 588-595. 
[10] Tejado A., Chen W. C., Alam M. N., Ven T. G. M v. d. 2014. Superhydrophobic Foam-like Cellulose Made of Hydrophobized Cellulose Fibres. Cellulose. 21: 1735-1743.

[11] Arkles B. 2006. Hydrophobicity, Hydrophilicity and Silanes. Paint and Coating Industry Magazine

[12] Lee, B.H. Choi, J.H. and Kim, H.J. 2006. Coating Performances and Characteristics for UV-Curable Aliphatic Urethane Acylates Coatings Containing Norrish Type I Photoinitiators. Journal of Coatings Technology and Research. 3(3): 221-229.

[13] Oribaya O., Feng X., Rempel G. L., Pan Q. 2017. Synthesis of Lignin-based Polyurethane/graphene Oxide Foam and Its Application as An Absorbent for Oil Spill Clean-ups and Recovery. Chemical Engineering Journal. 323: 191-202.

[14] Li S., Xie H., Zhang S., Wang X. 2007. Facile Transformation of Hydrophilic Cellulose Into Superhydrophobic Cellulose. The Royal Society of Chemistry. 4857-4859.

[15] Siqueira, R. L., Yoshida, I. V. P., Pardini, L.C., Shiavon, M. A. 2007. Poly (borosiloxanes) as Precursors for Carbon Fiber Ceramic Matrix Composites. Material Research. 10(2): 147-151

[16] Filiporic, R., Lazic, D., Perusic, M., Stijeporic, I. 2010. Oil Absorption in Mesoporous Silica Particles. Processing and Application of Ceramics. 4(4): 265-269

[17] Wang J., Zheng Y., Wang Q. 2013. Coated Kapok Fiber for Removal of Spilled Oil. Marine Pollution Bulletin. 69: 91-96. 